\title{
Certain inequalities for the modified Bessel-type function
}

\author{
Min-Jie Luo ${ }^{1 *}$ (D) and Ravinder Krishna Raina2,3
}

\section{"Correspondence:}

mathwinnie@live.com

'Department of Mathematics, East China Normal University, Shanghai, People's Republic of China Full list of author information is available at the end of the article

\begin{abstract}
We establish some new inequalities for the modified Bessel-type function $\lambda_{\nu, \sigma}^{(\beta)}(x)$ studied by Glaeske et al. [in J. Comput. Appl. Math. 118(1-2):151-168, 2000] as the kernel of an integral transformation that modifies Krätzel's integral transformation. The inequalities obtained are closely related to the generalized Hurwitz-Lerch zeta function and complementary incomplete gamma function. We also deduce some useful inequalities for the modified Bessel function of the second kind $K_{v}(x)$ and Mills' ratio $M(x)$ as worthwhile applications of our main results.
\end{abstract}

MSC: 26D15; 33B20; 33C10

Keywords: Čebyšev inequality; Generalized Hurwitz-Lerch zeta function; Hölder's inequality; Incomplete gamma function; Mills' ratio

\section{Introduction and motivation}

In a series of papers [2-4], E. Krätzel introduced and studied an integral transformation

$$
\mathcal{L}_{v}^{(n)}\{f\}(x)=\int_{0}^{\infty} \lambda_{v}^{(n)}\left(n(x t)^{1 / n}\right) f(t) \mathrm{d} t \quad\left(x \in \mathbb{R}_{+}\right)
$$

where $\lambda_{v}^{(n)}(x)$ is a Bessel-type function defined by

$$
\begin{gathered}
\lambda_{v}^{(n)}(x)=\frac{(2 \pi)^{(n-1) / 2} \sqrt{n}}{\Gamma\left(v+1-\frac{1}{n}\right)}\left(\frac{x}{n}\right)^{n v} \int_{1}^{\infty}\left(t^{n}-1\right)^{v-1 / n} \mathrm{e}^{-x t} \mathrm{~d} t \\
\left(v>\frac{1}{n}-1 ; n \in \mathbb{N}:=\{1,2, \ldots\}\right) .
\end{gathered}
$$

The integral transformation $\mathcal{L}_{v}^{(n)}$ reduces to some celebrated integral transformations by suitably specializing the parameters. For instance, when $n=1$, it reduces to the Laplace transformation $\mathcal{L}$, and when $n=2$, we have $\lambda_{v}^{(2)}(x)=2^{1-v} x^{v} K_{v}(x)$, where $K_{v}(x)$ is the modified Bessel function of the second kind of order $v$. The integral transformation (1) then becomes the Meijer transformation $\mathcal{K}_{v}$. Several authors have studied this integral transformation and its variants (see, for example, [5, 6] and [7]).

The kernel $\lambda_{v}^{(n)}(x)$ is of interest and particular significance from the point of view of the theory of special functions. In a recent work, R.E. Gaunt [8] established the following

(c) The Author(s) 2019. This article is distributed under the terms of the Creative Commons Attribution 4.0 International License (http://creativecommons.org/licenses/by/4.0/), which permits unrestricted use, distribution, and reproduction in any medium, provided you give appropriate credit to the original author(s) and the source, provide a link to the Creative Commons license, and indicate if changes were made. 
inequality for the function $\lambda_{v}^{(n)}(x)$ :

$$
\begin{aligned}
\lambda_{v}^{(n)}(x) & \geq \frac{1}{\sqrt{n}}\left(\frac{n}{n-1}\right)^{v+1-1 / n} P_{n, v}(x) Q_{n, n, v}(x) \mathrm{e}^{-x} \\
(0 & \left.\leq v \leq \frac{1}{n} ; n \in\{1,2,3, \ldots\}\right),
\end{aligned}
$$

where (for convenience)

$$
P_{r, s}(x):=(2 \pi)^{(r-1) / 2}\left(\frac{x}{r}\right)^{s r}
$$

and

$$
Q_{r, s, t}(x):=\frac{\Gamma\left(\frac{x}{r-1}+\frac{1}{s}-t\right)}{\Gamma\left(\frac{x}{r-1}+1\right)} .
$$

The equality in (2) holds if and only if $v=\frac{1}{n}$. If $v>\frac{1}{n}$, the strict inequality is reversed and holds for all $x>(n-1)\left(v-\frac{1}{n}\right)$. By setting $n=2$ in (2), Gaunt [8] obtained a lower bound for the modified Bessel function $K_{v}(x)$, that is,

$$
K_{v}(x) \geq \sqrt{\frac{\pi}{2}} \frac{\Gamma\left(x+\frac{1}{2}-v\right)}{\Gamma(x+1)} x^{v} \mathrm{e}^{-x} \quad\left(0 \leq v \leq \frac{1}{2}\right) .
$$

It is worth mentioning here that the lower bound in (5) is better than that of Luke mentioned in [9, p. 63, Eq. (6.28)] for $K_{v}(x)$ when $x$ is small (see [8, p. 990, Remark 1]). The lower bound for $K_{v}(x)$ in the case $v=0$ was also established by Gaunt [10]. Furthermore, inequality (2) was obtained actually by generalizing the approach there that Grant had adopted in [10].

In this paper, we establish some inequalities for the function $\lambda_{\nu, \sigma}^{(\beta)}(x)$ defined by

$$
\begin{gathered}
\lambda_{\nu, \sigma}^{(\beta)}(x)=\frac{\beta}{\Gamma\left(v+1-\frac{1}{\beta}\right)} \int_{1}^{\infty}\left(t^{\beta}-1\right)^{\nu-1 / \beta} t^{\sigma} \mathrm{e}^{-x t} \mathrm{~d} t \\
\left(\beta>0, \Re(v)>\frac{1}{\beta}-1, \sigma \in \mathbb{R}, x \in \mathbb{R}_{+}\right) .
\end{gathered}
$$

The function $\lambda_{v, \sigma}^{(\beta)}(x)$ was earlier introduced by Glaeske et al. [1] as the kernel of the modified Bessel-type integral transformation

$$
\mathcal{L}_{v, \sigma}^{(\beta)}\{f\}(x)=\int_{0}^{\infty} \lambda_{\nu, \sigma}^{(\beta)}(x t) f(t) \mathrm{d} t
$$

The integral transformation $\mathcal{L}_{v, \sigma}^{(\beta)}$ is in some sense a modification of the Krätzel's integral transformation $\mathcal{L}_{\nu}^{(n)}$ defined above by (1).

The functions $\lambda_{v, \sigma}^{(\beta)}(x)$ and $\lambda_{v}^{(n)}(x)$ are related through the formula

$$
P_{n, v}(x) \lambda_{v, 0}^{(n)}(x)=\sqrt{n} \lambda_{v}^{(n)}(x),
$$


where $P_{n, v}(x)$ is given by (3), and in the literature there are several papers devoted to the function $\lambda_{\nu, \sigma}^{(\beta)}(x)$ and to its related integral transformation $\mathcal{L}_{v, \sigma}^{(\beta)}$; see, for example, [11-14] and [15].

When all parameters and the variable are real, the asymptotic behavior of $\lambda_{\nu, \sigma}^{(\beta)}(x)$ is given by (see [14, p. 39]; see also [12])

$$
\lambda_{\nu, \sigma}^{(\beta)}(x) \sim \frac{\Gamma\left(-\nu-\frac{\sigma}{\beta}\right)}{\Gamma\left(1-\frac{\sigma+1}{\beta}\right)} \quad\left(\frac{1}{\beta}-1<\nu<-\frac{\sigma}{\beta}, \sigma \in \mathbb{R}, \beta>0 ; x \rightarrow 0^{+}\right)
$$

and

$$
\lambda_{\nu, \sigma}^{(\beta)}(x) \sim \beta^{\nu+1-1 / \beta} x^{(1 / \beta)-\nu-1} \mathrm{e}^{-x} \quad\left(v>1-\frac{1}{\beta}, \sigma \in \mathbb{R}, \beta>0 ; x \rightarrow \infty\right) .
$$

Our main results obtained in Sect. 3 involve the complementary incomplete gamma function $\Gamma(a, z)$ and the generalized Hurwitz-Lerch zeta function $\Phi_{\mu}^{*}(z, s, a)$. Their definitions and various properties are presented in Sect. 2.

\section{Definitions and auxiliary results}

In this section, we first briefly introduce the complementary incomplete gamma function and the generalized Hurwitz-Lerch zeta function, and establish for the latter a new property that plays an important role when we analyze the accuracy of the bounds obtained in Sect. 3. We also prove some auxiliary results which are required in the proofs of our main results.

The complementary incomplete gamma function $\Gamma(a, z)$ is defined by (see [16, p. 174]; see also [17, Chap. IX])

$$
\Gamma(a, z)=\int_{z}^{\infty} t^{a-1} \mathrm{e}^{-t} \mathrm{~d} t
$$

which by a simple change of variable and $a=\frac{1}{2}$ gives

$$
\Gamma\left(\frac{1}{2}, z^{2}\right)=\sqrt{\pi} \operatorname{erfc}(z)
$$

where $\operatorname{erfc}(z)$ is the complementary error function defined in [16, p. 160, Eq. (7.2.2)].

The generalized Hurwitz-Lerch zeta function $\Phi_{\mu}^{*}(z, s, a)$ was introduced in [18] (see also [19-21] and [22]) and is of the form:

$$
\begin{aligned}
\Phi_{\mu}^{*}(z, s, a) & =\sum_{n=0}^{\infty} \frac{(\mu)_{n}}{n !} \frac{z^{n}}{(a+n)^{s}} \\
(\mu \in \mathbb{C} ; a & \in \mathbb{C} \backslash\{0,-1,-2, \ldots\} ; s \in \mathbb{C} \text { when }|z|<1 ; \Re(s-\mu)>0 \text { when }|z|=1),
\end{aligned}
$$

and its integral representation is given by

$$
\begin{aligned}
& \Phi_{\mu}^{*}(z, s, a)=\frac{1}{\Gamma(s)} \int_{0}^{\infty} t^{s-1} \mathrm{e}^{-a t}\left(1-z \mathrm{e}^{-t}\right)^{-\mu} \mathrm{d} t \\
& \quad(\mu \in \mathbb{C} ; \Re(a)>0 ; \Re(s)>0 \text { when }|z|<1 ; \Re(s-\mu)>0 \text { when } z=1) .
\end{aligned}
$$


It is worth mentioning here that $\Phi_{\mu}^{*}(z, s, a)$ can be viewed as a Riemann-Liouville fractional derivative of the classical Hurwitz-Lerch function $\Phi(z, s, a)$ (see [23]; see also [22, p. 208] and [24]).

By setting $\mu=1$ in (10), we get the classical Hurwitz-Lerch zeta function $\Phi(z, s, a)$ (see [22, p. 194]). On the other hand, if we put $\mu=0$ in (11), then we find the following simple form:

$$
\Phi_{0}^{*}(z, s, a)=a^{-s}
$$

which is also used below. When $s=\ell \in \mathbb{N}$ in (10), we can make use of the simple relation that $a /(a+n)=(a)_{n} /(a+1)_{n}$ to obtain

$$
\begin{aligned}
& \Phi_{\mu}^{*}(z, \ell, a)=a^{-\ell}{ }_{\ell+1} F_{\ell}\left[\begin{array}{c}
\mu, a, \ldots, a \\
a+1, \ldots, a+1
\end{array} ; z\right. \\
& (\mu \in \mathbb{C} ; a \in \mathbb{C} \backslash\{0,-1,-2, \ldots\} \text { when }|z|<1 ; \Re(\ell-\mu)>0 \text { when }|z|=1) .
\end{aligned}
$$

If $|z|<1$, expression (13) gives immediately

$$
\Phi_{\mu}^{*}(z, \ell, a) \sim(1-z)^{-\mu} a^{-\ell}
$$

as $a \rightarrow+\infty$. But in Sect. 3 below, we shall use a different asymptotic formula for $\Phi_{\mu}^{*}(z, \ell, a)$ for large $a$ when $z=1$ and this formula cannot be determined from formula (14). We prefer here to examine a simpler case of determining the asymptotic behavior of the function defined by (10) and the result is included in the following proposition.

Proposition 2.1 For $0 \leq \mu<2$, we have

$$
\Phi_{\mu}^{*}(1,2, a) \sim \Gamma(2-\mu) a^{\mu-2}
$$

as $a \rightarrow+\infty$.

Proof From (13), we readily have

$$
\Phi_{\mu}^{*}(1,2, a)=a^{-2}{ }_{3} F_{2}\left[\begin{array}{c}
\mu, a, a \\
a+1, a+1
\end{array} ; 1\right]
$$

The problem is now converted into studying the asymptotics of the ${ }_{3} F_{2}$-function with four large parameters. Recall that (see [25, p. 246, Eq. (17)]; see also [26, p. 537, Eq. (49)])

$$
{ }_{3} F_{2}\left[\begin{array}{cc}
\mu, a, & a \\
a+1, & a+1
\end{array} ; 1\right]=\frac{a \Gamma(1-\mu) \Gamma(a+1)}{\Gamma(1+a-\mu)}[\psi(1+a-\mu)-\psi(a)]
$$

where $\Re(\mu)<2$ and $\psi(z)$ is the digamma function defined by (see [22, p. 24])

$$
\psi(z):=\frac{\mathrm{d}}{\mathrm{d} z} \ln \Gamma(z)=\frac{\Gamma^{\prime}(z)}{\Gamma(z)}
$$


It is well-known that (see [16, p. 140, Eq. (5.11.2)])

$$
\psi(z) \sim \ln z-\frac{1}{2 z}-\sum_{k=1}^{\infty} \frac{B_{2 k}}{2 k z^{2 k}}, \quad \text { as } z \rightarrow \infty \text { in }|\arg (z)| \leq \pi-\delta<\pi,
$$

where $B_{2 k}$ are the Bernoulli numbers, and also in view of [16, p. 141, Eq. (5.11.12)]:

$$
\frac{\Gamma(z+a)}{\Gamma(z+b)} \sim z^{a-b} \sum_{k=0}^{\infty} \frac{G_{k}(a, b)}{z^{k}}
$$

where $G_{0}(a, b)=1, G_{1}(a, b)=\frac{1}{2}(a-b)(a+b-1), \ldots$, can be expressed in terms of the generalized Bernoulli polynomials. Hence from (16), we find that

$$
{ }_{3} F_{2}\left[\begin{array}{cc}
\mu, a, & a \\
a+1, & a+1
\end{array} ; 1\right] \sim \Gamma(1-\mu) a^{1+\mu} \ln \left(1+\frac{1-\mu}{a}\right) \sim \Gamma(2-\mu) a^{\mu},
$$

as $a \rightarrow+\infty$.

We now prove the following useful lemma.

Lemma 2.2 Let $\rho>1$ and $\alpha, u \in \mathbb{R}_{+}$, then

$$
\frac{\rho}{\rho-1}\left(\mathrm{e}^{\alpha u}-1\right)>\left(1+\frac{\alpha u}{\rho-1}\right)^{\rho}-1 \text {. }
$$

Proof Let a function $f(u)$ be defined by

$$
f(u):=\mathrm{e}^{\alpha u}-1-\frac{\rho-1}{\rho}\left(1+\frac{\alpha u}{\rho-1}\right)^{\rho}+\frac{\rho-1}{\rho} .
$$

To prove inequality (18), it suffices to establish the positivity of the function $f$ when $\rho>1$ and $\alpha, u \in \mathbb{R}_{+}$. Since $f(0)=0$, we only need to show that

$$
f^{\prime}(u)=\alpha\left[\mathrm{e}^{\alpha u}-\left(1+\frac{\alpha u}{\rho-1}\right)^{\rho-1}\right]>0,
$$

which, however, follows directly from the elementary inequality:

$$
\left(1+\frac{x}{y}\right)^{y}<\mathrm{e}^{x} \quad(x>0, y>0) .
$$

Remark 2.3 A special case of (18) when $\alpha=2$ and $\rho$ is restricted to $\mathbb{N}$ was already proved by Gaunt [8, p. 989] using a different approach.

Let us now consider an integral of the form:

$$
\mathfrak{Z}(\alpha, \beta, \nu, x ; \sigma):=\int_{0}^{\infty}\left(\mathrm{e}^{\alpha u}-1\right)^{\nu-1 / \beta} u^{\sigma} \mathrm{e}^{-\alpha x u /(\beta-1)} \mathrm{d} u,
$$

where $\beta>1, x \in \mathbb{R}_{+}, \sigma \in \mathbb{R}$ and $0 \leq v \leq \frac{1}{\beta}$. We show below in Lemma 2.4 that this integral can be expressed in terms of the generalized Hurwitz-Lerch zeta function $\Phi_{\mu}^{*}(z, s, a)$ defined by (11). 
Lemma 2.4 Let $x \in \mathbb{R}_{+}, \beta>1,0 \leq v \leq \frac{1}{\beta}$ and $\sigma>\frac{1}{\beta}-v-1$. Then

$$
\mathfrak{Z}(\alpha, \beta, \nu, x ; \sigma)=\frac{\Gamma(\sigma+1)}{\alpha^{\sigma+1}} \Phi_{(1 / \beta)-\nu}^{*}\left(1, \sigma+1, \frac{x}{\beta-1}+\frac{1}{\beta}-v\right),
$$

where $\Phi_{\mu}^{*}(x, \alpha, a)$ is the generalized Hurwitz-Lerch zeta function defined by (11).

Furthermore, we have

$$
\mathfrak{Z}(\alpha, \beta, v, x ; 0)=\frac{1}{\alpha} \Gamma\left(v-\frac{1}{\beta}+1\right) Q_{\beta, \beta, v}(x),
$$

where $Q_{\beta, \beta, v}(x)$ is given by (4).

Proof Making a change of variable $\tau=\alpha u$, we obtain

$$
\mathfrak{Z}(\alpha, \beta, \nu, x ; \sigma)=\frac{1}{\alpha^{\sigma+1}} \int_{0}^{\infty} \tau^{\sigma}\left(1-\mathrm{e}^{-\tau}\right)^{\nu-1 / \beta} \mathrm{e}^{-(x /(\beta-1)+(1 / \beta)-\nu) \tau} \mathrm{d} \tau .
$$

When $\sigma+1>\frac{1}{\beta}-v$ and

$$
\frac{x}{\beta-1}+\frac{1}{\beta}-v>0
$$

the integral on the right-hand side of (23) converges and can be expressed in terms of the generalized Hurwitz-Lerch zeta function with the help of the integral representation (11) with $x=1$. Condition (24) can be removed because its equivalent form $x>(\beta-1)\left(v-\frac{1}{\beta}\right)$ always holds due to the assumption $x \in \mathbb{R}_{+}$. This proves the first assertion of the lemma.

When $\sigma=0$, the generalized Hurwitz-Lerch zeta function on the right-hand side of (21) remains well-defined and thus its integral representation can be applied to evaluate $\Phi_{(1 / \beta)-v}^{*}\left(1,1, \frac{x}{\beta-1}+\frac{1}{\beta}-v\right)$. We have then

$$
\begin{aligned}
\Phi_{(1 / \beta)-v}^{*}\left(1,1, \frac{x}{\beta-1}+\frac{1}{\beta}-v\right) & =\int_{0}^{\infty}\left(1-\mathrm{e}^{-\tau}\right)^{\nu-1 / \beta} \mathrm{e}^{-(x /(\beta-1)+(1 / \beta)-v) \tau} \mathrm{d} \tau \\
& =\Gamma\left(v-\frac{1}{\beta}+1\right) \frac{\Gamma\left(\frac{x}{\beta-1}+\frac{1}{\beta}-v\right)}{\Gamma\left(\frac{x}{\beta-1}+1\right)} .
\end{aligned}
$$

The desired second assertion of (22) now follows from (21) in conjunction with (4) and (25), and the proof is complete.

We shall need the following version of Čebyšev inequality [27, p. 40, Theorem 10]; see also $[28,29]$ and [30].

Lemma 2.5 Let $f$ and $g$ be two functions which are integrable and monotone in the same sense on $X:=(a, b)$ and let $\mu$ be a positive and integrable function on $X$. Then

$$
\int_{X} \mu(t) \mathrm{d} t \int_{X} f(t) g(t) \mu(t) \mathrm{d} t \geq \int_{X} f(t) \mu(t) \mathrm{d} t \int_{X} g(t) \mu(t) \mathrm{d} t,
$$

with equality if and only if one of the functions $f, g$ reduces to a constant.

Iff and $g$ are monotone in the opposite sense, inequality (26) reverses. 
Lu and Rain Journal of Inequalities and Applications

(2019) 2019:20

Page 7 of 15

We also need Hölder's inequality for $p \in(0,1)$ and $q \in(-\infty, 0)$. For $q \in(-\infty, 0)$, we define (see [31, p. 404, Definition 16.53])

$$
\|g\|_{q}:=\left(\int_{X}|g|^{q} \mathrm{~d} \mu\right)^{1 / q} \in[0, \infty]
$$

it is understood that if $\int_{X}|g|^{q} \mathrm{~d} \mu=\infty$, then $\|g\|_{q}=0$ and if $\int_{X}|g|^{q} \mathrm{~d} \mu=0$, then $\|g\|_{q}=\infty$.

Lemma 2.6 ([31, p. 404, Theorem 16.54]) Given a measure space $(X, \mathfrak{U}, \mu)$, let $p \in(0,1)$ and $q \in(-\infty, 0)$ be such that $\frac{1}{p}+\frac{1}{q}=1$. Let $f$ and $g$ be two extended complex-valued $\mathfrak{U}$ measurable functions on $X$ such that $|f|<\infty$ are. on $X$ and $0<|g|<\infty$ ae. on $X$ with $0<\|g\|_{q}<\infty$. Then

$$
\|f g\|_{1} \geq\|f\|_{p}\|g\|_{q} .
$$

If $0<\|f\|_{1}<\infty$ and $0<\|g\|_{q}<\infty$, then the equality in (27) holds if and only if

$$
A|f|^{p}=B|g|^{q} \quad \text { ae. on } X \text { for some } A, B>0 .
$$

3 Main results

Theorem 3.1 Let $x \in \mathbb{R}_{+}, \beta>1,0 \leq v \leq \frac{1}{\beta}, \sigma \in \mathbb{R}$ and let $\lambda_{\nu, \sigma}^{(\beta)}(x)$ be defined by (6).

(i) For $\sigma \geq 0$, we have

$$
\begin{aligned}
\lambda_{\nu, \sigma}^{(\beta)}(x) \geq & C_{\sigma}\left(\frac{\beta}{\beta-1}\right)^{v+1-1 / \beta} Q_{\beta, \beta, v}(x) \mathrm{e}^{-x}+\frac{C_{\sigma}}{(\beta-1)^{\sigma}}\left(\frac{\beta}{\beta-1}\right)^{v+1-1 / \beta} \\
& \cdot \frac{\Gamma(\sigma+1)}{\Gamma\left(v+1-\frac{1}{\beta}\right)} \Phi_{(1 / \beta)-v}^{*}\left(1, \sigma+1, \frac{x}{\beta-1}+\frac{1}{\beta}-v\right) \mathrm{e}^{-x}
\end{aligned}
$$

where $\Phi_{\mu}^{*}(z, s, a)$ is given by (11) and $C_{\sigma}$ is given below by (31).

(ii) For $\sigma<0$, we have

$$
\lambda_{\nu, \sigma}^{(\beta)}(x)>\left(\frac{\beta}{\beta-1}\right)^{v+1-1 / \beta} Q_{\beta, \beta, v}(x-\sigma) \mathrm{e}^{-x}
$$

where $Q_{\beta, \beta, \nu}(x)$ is given by (4).

Proof Setting $t=\frac{\alpha u}{\beta-1}+1$ in (6), we find that

$$
\begin{aligned}
\lambda_{\nu, \sigma}^{(\beta)}(x)= & \frac{\alpha}{\Gamma\left(\nu+1-\frac{1}{\beta}\right)} \frac{\beta}{\beta-1} e^{-x} \\
& \cdot \int_{0}^{\infty}\left[\left(\frac{\alpha u}{\beta-1}+1\right)^{\beta}-1\right]^{\nu-1 / \beta}\left(\frac{\alpha u}{\beta-1}+1\right)^{\sigma} \mathrm{e}^{-\alpha x u /(\beta-1)} \mathrm{d} u .
\end{aligned}
$$

When $v-\frac{1}{\beta} \leq 0$, we have by Lemma 2.2,

$$
\left[\left(\frac{\alpha}{\beta-1} u+1\right)^{\beta}-1\right]^{\nu-1 / \beta} \geq\left(\frac{\beta}{\beta-1}\right)^{\nu-1 / \beta}\left(\mathrm{e}^{\alpha u}-1\right)^{\nu-1 / \beta}
$$


Thus, for $0 \leq v \leq \frac{1}{\beta}$, we obtain the following inequality:

$$
\lambda_{\nu, \sigma}^{(\beta)}(x) \geq \frac{\alpha}{\Gamma\left(\nu+1-\frac{1}{\beta}\right)}\left(\frac{\beta}{\beta-1}\right)^{\nu+1-1 / \beta} \mathfrak{I}(\alpha, \beta, \nu, x ; \sigma) \mathrm{e}^{-x},
$$

where $\mathfrak{I}(\alpha, \beta, v, x ; \sigma)$ is defined by

$$
\mathfrak{I}(\alpha, \beta, \nu, x ; \sigma):=\int_{0}^{\infty}\left(e^{\alpha u}-1\right)^{\nu-1 / \beta}\left(\frac{\alpha u}{\beta-1}+1\right)^{\sigma} \mathrm{e}^{-\alpha x u /(\beta-1)} \mathrm{d} u .
$$

In order to estimate a lower bound of $\mathfrak{I}(\alpha, \beta, \nu, x ; \sigma)$, we need to take into account the cases $\sigma \geq 0$ and $\sigma<0$, which are considered as follows:

Case 1: $\sigma \geq 0$. Let us recall that for $a, b \in \mathbb{R}_{+}$, we have $(a+b)^{\sigma} \geq C_{\sigma}\left(a^{\sigma}+b^{\sigma}\right)$, where $C_{\sigma}$ is defined by

$$
C_{\sigma}:= \begin{cases}1, & \sigma \geq 1 \\ 2^{\sigma-1}, & 0 \leq \sigma<1\end{cases}
$$

Thus, for the case $\sigma \geq 0$, we have

$$
\left(\frac{\alpha u}{\beta-1}+1\right)^{\sigma} \geq C_{\sigma}\left[1+\left(\frac{\alpha}{\beta-1}\right)^{\sigma} u^{\sigma}\right]
$$

Therefore,

$$
\mathfrak{I}(\alpha, \beta, x ; \sigma) \geq C_{\sigma} \Im(\alpha, \beta, \nu, x ; 0)+C_{\sigma}\left(\frac{\alpha}{\beta-1}\right)^{\sigma} \mathfrak{I}(\alpha, \beta, \nu, x ; \sigma),
$$

where $\mathfrak{Z}(\alpha, \beta, \nu, x ; \sigma)$ is the integral defined in (20) and

$$
\Im(\alpha, \beta, \nu, x ; 0)=\mathfrak{Z}(\alpha, \beta, v, x ; 0)=\frac{1}{\alpha} \Gamma\left(v-\frac{1}{\beta}+1\right) Q_{\beta, \beta, v}(x) .
$$

Hence, we have

$$
\begin{aligned}
& \Im(\alpha, \beta, v, x ; \sigma) \geq \frac{C_{\sigma}}{\alpha} \Gamma\left(v-\frac{1}{\beta}+1\right) Q_{\beta, \beta, v}(x) \\
&+\frac{C_{\sigma}}{\alpha} \frac{\Gamma(\sigma+1)}{(\beta-1)^{\sigma}} \Phi_{(1 / \beta)-v}^{*}\left(1, \sigma+1, \frac{x}{\beta-1}+\frac{1}{\beta}-v\right) \\
&\left(x \in \mathbb{R}_{+}, \beta>1,0 \leq v \leq \frac{1}{\beta} ; \sigma \geq 0\right) .
\end{aligned}
$$

Combining this inequality with (30), we get the first inequality of Theorem 3.1.

Case 2: $\sigma<0$. By using inequality (19), we readily get

$$
\left(\frac{\alpha u}{\beta-1}+1\right)^{\sigma}>\mathrm{e}^{\alpha \sigma u /(\beta-1)}
$$


and thus

$$
\begin{gathered}
\Im(\alpha, \beta, v, x ; \sigma)>\frac{1}{\alpha} \Gamma\left(v-\frac{1}{\beta}+1\right) Q_{\beta, \beta, v}(x-\sigma) \\
\left(x \in \mathbb{R}_{+}, \beta>1,0 \leq v \leq \frac{1}{\beta} ; \sigma<0\right) .
\end{gathered}
$$

Now applying (32) to (30), we obtain the second inequality of Theorem 3.1.

Corollary 3.2 Let $x \in \mathbb{R}_{+}$and $0 \leq v \leq \frac{1}{2}$, then

$$
K_{v+1}(x) \geq \sqrt{\frac{\pi}{2}}\left[\frac{\Gamma\left(x+\frac{1}{2}-v\right)}{\Gamma(x+1)}+\frac{\Phi_{(1 / 2)-v}^{*}\left(1,2, x+\frac{1}{2}-v\right)}{\Gamma\left(v+\frac{1}{2}\right)}\right] x^{v} \mathrm{e}^{-x}
$$

Proof Let $\beta=2$ and $\sigma=1$ in (28), then we get

$$
\lambda_{v, 1}^{(2)}(x) \geq 2^{v+1 / 2}\left[Q_{2,2, v}(x)+\frac{\Phi_{(1 / 2)-v}^{*}\left(1,2, x+\frac{1}{2}-v\right)}{\Gamma\left(v+\frac{1}{2}\right)}\right] \mathrm{e}^{-x} .
$$

It is known for $v \in \mathbb{C}\left(\Re(v)>-\frac{1}{2}\right)$ and $z \in \mathbb{C}(\Re(z)>0)$ that (see [11, p. 97, Eq. (3.3)])

$$
\lambda_{v, 1}^{(2)}(z)=2^{v+1} \pi^{-1 / 2} z^{-v} K_{-(v+1)}(z) .
$$

Using (34) and the fact that $K_{v}(z)=K_{-v}(z)$, we get inequality (33).

Remark 3.3 Let us denote the lower bound in (33) by $\mathrm{D}_{v}(x)$. Then for $v=\frac{1}{2}$ and using (12), we have

$$
\begin{aligned}
\mathrm{D}_{1 / 2}(x) & =\sqrt{\frac{\pi}{2}}\left[\frac{\Gamma(x)}{\Gamma(x+1)}+\Phi_{0}^{*}(1,2, x)\right] x^{1 / 2} \mathrm{e}^{-x}=\sqrt{\frac{\pi}{2}}\left(\frac{1}{x}+\frac{1}{x^{2}}\right) x^{1 / 2} \mathrm{e}^{-x} \\
& =\sqrt{\frac{\pi}{2}}(1+x) x^{-3 / 2} \mathrm{e}^{-x}=K_{3 / 2}(x),
\end{aligned}
$$

which means the equality in (33) holds when $v=\frac{1}{2}$. For $v \in\left[0, \frac{1}{2}\right.$ ), we have from Proposition 2.1 that

$$
\mathrm{D}_{v}(x) \sim \sqrt{\frac{\pi}{2}} x^{-1 / 2} \mathrm{e}^{-x}+\sqrt{\frac{\pi}{2}} \frac{\Gamma\left(v+\frac{3}{2}\right)}{\Gamma\left(v+\frac{1}{2}\right)} x^{-3 / 2} \mathrm{e}^{-x},
$$

as $x \rightarrow \infty$. The second term in (35) can be omitted. Recall that (see [16, p. 249, Eq. (10.25.3)])

$$
K_{v}(x) \sim \sqrt{\frac{\pi}{2 x}} \mathrm{e}^{-x}, \quad \text { as } x \rightarrow \infty,
$$

and we have for $v \in\left[0, \frac{1}{2}\right): D_{v}(x) \sim K_{v+1}(x)$ as $x \rightarrow \infty$. When $v \in\left(0, \frac{1}{2}\right)$, we always have $\lim _{x \rightarrow 0^{+}} D_{v}(x)=0$. But for $v=0$, we have

$$
\lim _{x \rightarrow 0^{+}} \mathrm{D}_{0}(x)=\frac{\pi \sqrt{2}}{2}+\frac{\sqrt{2}}{2} \Phi_{1 / 2}^{*}\left(1,2, \frac{1}{2}\right),
$$


which is finite. Note that the lower bound $D_{v}(x)$ does not have a singularity for $v \in\left[0, \frac{1}{2}\right)$ while, in view of [16, p. 252, Eqs. (10.30.2) and (10.30.3)]

$$
K_{v}(x) \sim \begin{cases}2^{v-1} \Gamma(v) x^{-v}, & x \rightarrow 0, \Re(v)>0, \\ -\ln x, & x \rightarrow 0, v=0\end{cases}
$$

we know $K_{v}(x)$ has a singularity at $x=0$. The limiting form (36) is of particular interest because its exact value is easily obtainable. Using (13) and (16), we have

$$
\Phi_{1 / 2}^{*}\left(1,2, \frac{1}{2}\right)=4_{3} F_{2}\left[\begin{array}{c}
\frac{1}{2}, \frac{1}{2}, \frac{1}{2} \\
\frac{3}{2}, \frac{3}{2}
\end{array} ;\right]=\left[\Gamma\left(\frac{1}{2}\right)\right]^{2}\left[\psi(1)-\psi\left(\frac{1}{2}\right)\right] .
$$

Since $\psi(1)=-\gamma$ and $\psi\left(\frac{1}{2}\right)=-\gamma-2 \ln 2$, we obtain

$$
\Phi_{1 / 2}^{*}\left(1,2, \frac{1}{2}\right)=2 \pi \ln 2,
$$

and therefore,

$$
\lim _{x \rightarrow 0^{+}} D_{0}(x)=\frac{\pi \sqrt{2}}{2}+\sqrt{2} \pi \ln 2 .
$$

The value of ${ }_{3} F_{2}[1]$ appearing in (37) can also be evaluated by using the following formula (see [32, p. 592, Eq. (24)]):

$$
{ }_{3} F_{2}\left[\begin{array}{c}
\frac{1}{2}, \frac{1}{2}, \frac{1}{2} \\
\frac{3}{2}, \frac{3}{2}
\end{array} ; z\right]=\frac{1}{2 \sqrt{z}} \mathrm{Cl}_{2}(2 \arcsin \sqrt{z})+\frac{\arcsin \sqrt{z}}{\sqrt{z}} \ln (2 \sqrt{z}),
$$

where $\mathrm{Cl}_{2}(\theta)$ is the Clausen integral (or function) defined by (see [22, p. 182, Eq. (45)])

$$
\mathrm{Cl}_{2}(\theta):=\sum_{n=1}^{\infty} \frac{\sin (n \theta)}{n^{2}}=-\int_{0}^{\theta} \ln \left[2 \sin \left(\frac{t}{2}\right)\right] \mathrm{d} t
$$

satisfying the property that $\mathrm{Cl}_{2}(n \pi)=0(n \in \mathbb{Z})($ see [22, p. 182, Eq. (49)]).

Theorem 3.4 Let $x \in \mathbb{R}_{+}, \beta>1,0 \leq v \leq \frac{1}{\beta}$ and $\sigma \leq 0$, then

$$
\lambda_{v, \sigma}^{(\beta)}(x) \geq\left(\frac{\beta}{\beta-1}\right)^{v+1-1 / \beta} Q_{\beta, \beta, v}(x) \Gamma(\sigma+1, x) x^{-\sigma}
$$

where $Q_{\beta, \beta, v}(x)$ is given by (4).

Proof Let $f(t):=\left(t^{\beta}-1\right)^{\nu-1 / \beta}$ and $g(t):=t^{\sigma}$. Since $v-\frac{1}{\beta} \leq 0$ and $\sigma \leq 0, f$ and $g$ are both decreasing (i.e., monotone in the same sense) on $(1, \infty)$, and thus Čebyšev inequality (26) with $\mu(t)=\mathrm{e}^{-x t}$ and $X=(1, \infty)$ is applicable to get the following result:

$$
\int_{1}^{\infty}\left(t^{\beta}-1\right)^{\nu-1 / \beta} t^{\sigma} \mathrm{e}^{-x t} \mathrm{~d} t
$$




$$
\begin{aligned}
& \geq\left(\int_{1}^{\infty} \mathrm{e}^{-x t} \mathrm{~d} t\right)^{-1} \int_{1}^{\infty}\left(t^{\beta}-1\right)^{\nu-1 / \beta} \mathrm{e}^{-x t} \mathrm{~d} t \int_{1}^{\infty} t^{\sigma} \mathrm{e}^{-x t} \mathrm{~d} t \\
& =\mathrm{e}^{x} x^{-\sigma} \Gamma(\sigma+1, x) \int_{1}^{\infty}\left(t^{\beta}-1\right)^{\nu-1 / \beta} \mathrm{e}^{-x t} \mathrm{~d} t .
\end{aligned}
$$

By using (6), we obtain that

$$
\lambda_{\nu, \sigma}^{(\beta)}(x) \geq \mathrm{e}^{x} x^{-\sigma} \Gamma(\sigma+1, x) \lambda_{\nu, 0}^{(\beta)}(x)
$$

For the Bessel-type function $\lambda_{v, 0}^{(\beta)}(x)$, by putting $\sigma=0$ in (28) and making use of formula (25) and relation (31), we infer that

$$
\lambda_{\nu, 0}^{(\beta)}(x) \geq\left(\frac{\beta}{\beta-1}\right)^{v+1-1 / \beta} Q_{\beta, \beta, v}(x) \mathrm{e}^{-x} \quad\left(x \in \mathbb{R}_{+}, \beta>1,0 \leq \nu \leq \frac{1}{\beta}\right) .
$$

Combining now (40) with (41), we get the desired inequality (39).

Remark 3.5 Let us put

$$
\mathrm{B}_{2}(x)=\left(\frac{\beta}{\beta-1}\right)^{v+1-1 / \beta} Q_{\beta, \beta, v}(x) \Gamma(\sigma+1, x) x^{-\sigma} .
$$

In view of [16, p. 179, Eq. (8.11.2)], we have

$$
\Gamma(\sigma+1, x) x^{-\sigma} \sim \mathrm{e}^{-x} \quad(x \rightarrow \infty) .
$$

Applying the familiar Stirling formula [16, p. 141, Eq. (5.11.7)], we find that

$$
Q_{\beta, \beta, v}(x) \sim(\beta-1)^{\nu+1-1 / \beta} x^{(1 / \beta)-\nu-1} \quad(x \rightarrow \infty),
$$

and upon using now (42) and (43), together with the above mentioned expression for $\mathrm{B}_{2}(x)$, we get

$$
\mathrm{B}_{2}(x) \sim \beta^{\nu+1-1 / \beta} x^{(1 / \beta)-\nu-1} \mathrm{e}^{-x} \quad(x \rightarrow \infty) .
$$

If we denote the lower bound in (29) by $B_{1}(x)$, then, by following the same arguments as described above for $\mathrm{B}_{2}(x)$, we obtain

$$
\mathrm{B}_{1}(x) \sim \beta^{\nu+1-1 / \beta} x^{(1 / \beta)-\nu-1} \mathrm{e}^{-x} \quad(x \rightarrow \infty) .
$$

Therefore, when $\sigma \leq 0$, we have $\mathrm{B}_{2}(x) \sim \mathrm{B}_{1}(x)(x \rightarrow \infty)$ and, in view of (7), we also know that both $\mathrm{B}_{1}(x)$ and $\mathrm{B}_{2}(x)$ have the same behavior as $\lambda_{\nu, \sigma}^{(\beta)}(x)$ at infinity. Now we consider the case when $x \rightarrow 0^{+}$. For $B_{2}$, we have

$$
\lim _{x \rightarrow 0^{+}} \mathrm{B}_{2}(x)=\left(\frac{\beta}{\beta-1}\right)^{v+1-1 / \beta} \frac{\Gamma\left(-v-\frac{\sigma}{\beta-1}+\frac{1}{\beta}\right)}{\Gamma\left(1-\frac{\sigma}{\beta-1}\right)} .
$$


In order to find the asymptotic behavior of $\mathrm{B}_{1}(x)$ as $x \rightarrow 0^{+}$, we only need to study the behavior of $\Gamma(\sigma+1, x) x^{-\sigma}$, which follows immediately from the fact that

$$
\Gamma(\sigma+1, x) \sim \Gamma(\sigma+1)-\frac{x^{\sigma+1}}{\sigma+1}[1+\mathcal{O}(x)],
$$

as $x \rightarrow 0^{+}$.

We observe that, when $\sigma>0$, the functions $f$ and $g$ defined in the proof of Theorem 3.4 are monotone in the opposite sense. We cannot therefore use Čebyšev inequality to find a lower bound for this case. However, the use of Hölder inequality (27) enables us to unify the cases $\sigma>0$ and $\sigma \leq 0$ more efficiently.

Theorem 3.6 Let $x \in \mathbb{R}_{+}, \beta>1,0 \leq v \leq \frac{1}{\beta}$ and $\sigma \in \mathbb{R}$. If $p \in(0,1)$ and $q \in(-\infty, 0)$ are such that $\frac{1}{p}+\frac{1}{q}=1$, then

$$
\begin{aligned}
\lambda_{\nu, \sigma}^{(\beta)}(x) \geq & \beta^{1 / q}\left(\frac{\beta}{\beta-1}\right)^{\nu-(1 / \beta)+1 / p} \frac{\left[\Gamma\left(\nu p-\frac{p}{\beta}+1\right)\right]^{1 / p}}{\Gamma\left(\nu+1-\frac{1}{\beta}\right)} \\
& \cdot\left[Q_{\beta, \beta / p, v p}(x)\right]^{1 / p}[\Gamma(\sigma q+1, x)]^{1 / q} x^{-\sigma-1 / q} \mathrm{e}^{-x / p},
\end{aligned}
$$

where $Q_{\beta, \beta / p, v p}(x)$ and $\Gamma(\alpha, x)$ are respectively defined by (4) and (8). The equality in (44) holds if and only if $v=\frac{1}{\beta}$ and $\sigma=0$.

Proof Let $f(t):=\left(t^{\beta}-1\right)^{\nu-1 / \beta} \mathrm{e}^{-x t / p}$ and $g(t):=t^{\sigma} \mathrm{e}^{-x t / q}$, then we have

$$
\begin{aligned}
\|f\|_{p} & =\frac{1}{\beta^{1 / p}}\left[\Gamma\left(v p-\frac{p}{\beta}+1\right) \lambda_{v p-(p / \beta)+1 / \beta, 0}^{(\beta)}(x)\right]^{1 / p}, \\
\|g\|_{q} & =x^{-\sigma-1 / q}[\Gamma(\sigma q+1, x)]^{1 / q}
\end{aligned}
$$

and $\|f g\|_{1}=\frac{1}{\beta} \Gamma\left(v+1-\frac{1}{\beta}\right) \lambda_{\nu, \sigma}^{(\beta)}(x)$, where $x \in \mathbb{R}_{+}, \sigma \in \mathbb{R}, \beta>1$ and $0 \leq v \leq \frac{1}{\beta}$.

Using Hölder's inequality (27), we have

$$
\lambda_{\nu, \sigma}^{(\beta)}(x) \geq \beta^{1 / q} \frac{\left[\Gamma\left(\nu p-\frac{p}{\beta}+1\right)\right]^{1 / p}}{\Gamma\left(v+1-\frac{1}{\beta}\right)}\left[\lambda_{v p-(p / \beta)+1 / \beta, 0}^{(\beta)}(x)\right]^{1 / p}[\Gamma(\sigma q+1, x)]^{1 / q} x^{-\sigma-1 / q} .
$$

Letting $v \rightarrow v p-\frac{p}{\beta}+\frac{1}{\beta}$ in inequality (41), which does not change the range of parameters as stated in the theorem, we obtain

$$
\begin{aligned}
& \lambda_{v p-(p / \beta)+1 / \beta, 0}^{(\beta)}(x)>\left(\frac{\beta}{\beta-1}\right)^{v p-(p / \beta)+1} Q_{\beta, \beta / p, v p}(x) \mathrm{e}^{-x} \\
& \left(x \in \mathbb{R}_{+}, \beta>1,0 \leq v \leq \frac{1}{\beta}\right) .
\end{aligned}
$$

Substituting inequality (46) into (45), we arrive at the desired inequality (44).

Corollary 3.7 Let $x \in \mathbb{R}_{+}$and $0 \leq v \leq \frac{1}{2}$. Then we have

$$
K_{v+1}(x)>\frac{1}{\sqrt{2 \pi}} \frac{\left[\Gamma\left(\frac{v}{3}+\frac{5}{6}\right) Q_{2,6, v / 3}(x)\right]^{3}}{\Gamma\left(v+\frac{1}{2}\right)[\operatorname{erfc}(\sqrt{x})]^{2}} x^{v+1} \mathrm{e}^{-3 x} .
$$


Proof Letting $\beta=2$ and $\sigma=1$ and $q=-\frac{1}{2}\left(p=\frac{1}{3}\right)$ in (44), we get

$$
\lambda_{v, 1}^{(2)}(x)>\frac{2^{v+1 / 2}}{\pi} \frac{\left[\Gamma\left(\frac{v}{3}+\frac{5}{6}\right) Q_{2,6, v / 3}(x)\right]^{3}}{\Gamma\left(v+\frac{1}{2}\right)[\operatorname{erfc}(\sqrt{x})]^{2}} x \mathrm{e}^{-3 x},
$$

where we have used relation (9). Now using (34) and the fact that $K_{v}(z)=K_{-v}(z)$, we get inequality (47).

Remark 3.8 If we denote the lower bound in (47) by $D_{v}^{*}(x)$, then, by using the fact that (see $[16$, p. 164, Eq. (7.12.1)])

$$
\operatorname{erfc}(x) \sim \frac{1}{\sqrt{\pi} x} \mathrm{e}^{-x^{2}}, \quad \text { as } x \rightarrow \infty,
$$

and the asymptotic behavior for the ratio of two gamma functions [16, p. 141, Eq. (5.11.12)] for the function $Q_{2,6, v / 3}(x)$ involved in $D_{v}^{*}(x)$ and defined above by (4), we have, taking $x \rightarrow \infty$ and after elementary calculations, the following:

$$
\mathrm{D}_{v}^{*}(x) \sim \mathfrak{c}(v) \sqrt{\frac{\pi}{2 x}} \mathrm{e}^{-x}, \quad \text { where } \mathfrak{c}(v):=\frac{\left[\Gamma\left(\frac{v}{3}+\frac{5}{6}\right)\right]^{3}}{\Gamma\left(v+\frac{1}{2}\right)}
$$

If we take $v=\frac{1}{2}$, then $\mathfrak{c}\left(\frac{1}{2}\right)=1$ and $D_{1 / 2}^{*}(x)$ has the same behavior as the modified Bessel function $K_{v+1}(x)$ at infinity. Further, we observe the property that $0<\mathfrak{c}(v) \leq 1$ for $v \in\left[0, \frac{1}{2}\right]$. To verify this property for function $\mathfrak{c}(v)$, we apply the logarithmic derivative to function $\mathfrak{c}(v)$ with respect to $v$ to get

$$
\frac{\mathrm{d}}{\mathrm{d} \nu} \ln \mathfrak{c}(v)=3 \frac{\mathrm{d}}{\mathrm{d} v} \ln \Gamma\left(\frac{v}{3}+\frac{5}{6}\right)-\frac{\mathrm{d}}{\mathrm{d} \nu} \ln \Gamma\left(v+\frac{1}{2}\right)=\psi\left(\frac{v}{3}+\frac{5}{6}\right)-\psi\left(v+\frac{1}{2}\right) .
$$

The digamma function $\psi(x)$ is increasing on $\mathbb{R}_{+}$and $\left(\frac{v}{3}+\frac{5}{6}\right)-\left(v+\frac{1}{2}\right)=\frac{1}{3}(1-2 v) \geq 0$ for $v \in\left[0, \frac{1}{2}\right]$, therefore $\frac{\mathrm{d}}{\mathrm{d} v} \ln \mathfrak{c}(v) \geq 0$, and thus we conclude that $\mathfrak{c}(v)$ is increasing on $\left[0, \frac{1}{2}\right]$ with 1 as its maximum. On the other hand, for $v=\frac{1}{2}$, we have

$$
\mathrm{D}_{1 / 2}^{*}(x)=\frac{1}{\sqrt{2 \pi}} \frac{x^{-3 / 2} \mathrm{e}^{-3 x}}{[\operatorname{erfc}(\sqrt{x})]^{2}},
$$

which implies that

$$
\mathrm{D}_{1 / 2}^{*}(x) \sim \frac{x^{-3 / 2}}{\sqrt{2 \pi}}, \quad \text { as } x \rightarrow 0^{+} .
$$

Moreover, for $v \in\left[0, \frac{1}{2}\right)$, we note that

$$
\mathrm{D}_{\nu}^{*}(x) \sim \frac{\mathfrak{c}(v)}{\sqrt{2 \pi}}\left[\Gamma\left(\frac{1}{6}-\frac{v}{3}\right)\right]^{3} x^{\nu+1}, \quad \text { as } x \rightarrow 0^{+} .
$$

Let us close this paper with an application of Corollary 3.7, leading to an interesting inequality for the Mills' ratio $\mathrm{M}(x)$ defined by (see [16, p. 163, Eq. (7.8.1)])

$$
\mathrm{M}(x):=\mathrm{e}^{x^{2}} \int_{x}^{\infty} \mathrm{e}^{-t^{2}} \mathrm{~d} t
$$


The Mills' ratio can be expressed using the complementary error function $\operatorname{erfc}(x)$ defined in (9) as

$$
M(x)=\frac{\sqrt{\pi}}{2} \mathrm{e}^{x^{2}} \operatorname{erfc}(x) .
$$

The study of inequalities involving Mills' ratio $M(x)$ and other related functions has a rich literature. The interesting reader may refer to [33-36].

Corollary 3.9 For $x \geq 0$, we have

$$
M(x)>\frac{1}{2 \sqrt{1+x^{2}}} .
$$

Proof By putting $v=\frac{1}{2}$ in (47), we get

$$
\frac{1}{\sqrt{2 \pi}}[\operatorname{erfc}(\sqrt{x})]^{-2} x^{-3 / 2} \mathrm{e}^{-3 x}<K_{3 / 2}(x)=\sqrt{\frac{\pi}{2}} x^{-3 / 2} \mathrm{e}^{-x}(1+x),
$$

which in turn gives a new inequality for $\operatorname{erfc}(x)$, that is,

$$
\operatorname{erfc}(x)>\frac{\mathrm{e}^{-x^{2}}}{\sqrt{\pi\left(1+x^{2}\right)}}
$$

Inequality (49) now follows from (48).

\section{Acknowledgements}

The authors would like to thank the referees and Professor Feng Qi (Editor, Journal of Inequalities and Applications) for their valuable comments and suggestions.

Funding

Not applicable.

Availability of data and materials

Not applicable.

Competing interests

The authors declare that they have no competing interests.

\section{Authors' contributions}

All authors contributed equally to the manuscript. All authors read and approved the final manuscript.

\section{Author details}

'Department of Mathematics, East China Normal University, Shanghai, People's Republic of China. ${ }^{2}$ M.P. University of Agriculture and Technology, Udaipur, India. ${ }^{3}$ Present address: Independent Consultant, Udaipur, India.

\section{Publisher's Note}

Springer Nature remains neutral with regard to jurisdictional claims in published maps and institutional affiliations.

Received: 10 August 2018 Accepted: 15 January 2019 Published online: 24 January 2019

\section{References}

1. Glaeske, H.J., Kilbas, A.A., Saigo, M.: A modified Bessel-type integral transform and its compositions with fractional calculus operators on spaces $\mathcal{F}_{p, \mu}$ and $\mathcal{F}_{p, \mu}^{\prime}$. J. Comput. Appl. Math. 118(1-2), 151-168 (2000)

2. Krätzel, E.: Eine verallgemeinerung der Laplace und Meijer tranformation. Wiss. Z. Univ. Jena Math. Naturwiss. Reihe 5 369-381 (1965)

3. Krätzel, E.: Übertragung der Post-Widderschen umkehrformel der Laplace-transformation auf die $\mathcal{L}$-transformation Math. Nachr. 35, 295-304 (1967) 
4. Krätzel, E.: Differentiations Sätze der $\mathcal{L}$-transformation und Differentialgleichungen nach dem Operator $\frac{\mathrm{d}}{\mathrm{d} t}\left[t^{\frac{1}{n}-v}\left(t^{1-\frac{1}{n}} \frac{\mathrm{d}}{\mathrm{d} t}\right)^{n} t^{v+1-\frac{2}{n}}\right]$. Math. Nachr. 35, 105-114 (1967)

5. Barrios, J.A., Betancor, J.J.: A Krätzel's integral transformation of distributions. Collect. Math. 42(1), 11-32 (1991)

6. Betancor, J.J., Barrios, J.A.: A real inversion formula for the Krätzel's generalized Laplace transform. Extr. Math. 6(2), 55-57 (1991)

7. Rao, G.L.N., Debnath, L.: A generalized Meijer transformation. Int. J. Math. Math. Sci. 8(2), 359-365 (1985)

8. Gaunt, R.E.: Inequalities for the modified Bessel function of the second kind and the kernel of the Krätzel integral transformation. Math. Inequal. Appl. 20(4), 987-990 (2017)

9. Luke, Y.L.: Inequalities for generalized hypergeometric functions. J. Approx. Theory 5, 41-65 (1972)

10. Gaunt, R.E.: Inequalities for modified Bessel functions and their integrals. J. Math. Anal. Appl. 420, 373-386 (2014)

11. Bonilla, B., Kilbas, A.A., Rivero, M., Rodriguez, L., Trujillo, J.J.: Modified Bessel-type function and solution of differential and integral equations. Indian J. Pure Appl. Math. 31(1), 93-109 (2000)

12. Kilbas, A.A., Rodríguez, L., Trujillo, J.J.: Asymptotic representations for hypergeometric-Bessel type function and fractional integrals. J. Comput. Appl. Math. 149, 469-487 (2002)

13. Kilbas, A.A., Saigo, M.: H-Transforms: Theory and Application. CRC Press, Boca Raton (2004)

14. Kilbas, A.A., Srivastava, H.M., Trujillo, J.J.: Theory and Applications of Fractional Differential Equations. North-Holland Mathematics Studies, vol. 204. Elsevier, Amsterdam (2006)

15. Kilbas, A.A., Trujillo, J.J.: Computation of fractional integrals via functions of hypergeometric and Bessel type. J. Comput. Appl. Math. 118(1-2), 223-239 (2000)

16. Olver, F.W.J., Lozier, D.W., Boisvert, R.F., Clark, C.W. (eds.): NIST Handbook of Mathematical Functions. Cambridge University Press, New York (2010)

17. Erdélyi, A., Magnus, W., Oberhettinger, F., Tricomi, F.: Higher Transcendental Functions, vol. 2. McGraw-Hill, New York (1953)

18. Goyal, S.P., Laddha, R.K.: On the generalized Riemann zeta functions and the generalized Lambert transform. Ganita Sandesh 11,99-108 (1997)

19. Luo, M.J., Raina, R.K.: Some new results related to a class of generalized Hurwitz zeta function. J. Class. Anal. 6(2), 103-112 (2015)

20. Luo, M.J., Parmar, R.K., Raina, R.K.: On extended Hurwitz-Lerch zeta function. J. Math. Anal. Appl. 448, 1281-1304 (2017)

21. Raina, R.K. Chhajed, P.K.: Certain results involving a class of functions associated with the Hurwitz Zeta function. Acta Math. Univ. Comen. 73, 89-100 (2004)

22. Srivastava, H.M., Choi, J.: Zeta and q-Zeta Functions and Associated Series and Integrals. Elsevier, Amsterdam (2012)

23. Lin, S.D., Srivastava, H.M.: Some families of the Hurwitz-Lerch Zeta functions and associated fractional derivative and other integral representations. Appl. Math. Comput. 154, 725-733 (2004)

24. Srivastava, H.M.: A new family of the $\lambda$-generalized Hurwitz-Lerch zeta functions with applications. Appl. Math. Inf. Sci. 8, 1485-1500 (2014)

25. Rao, K.S., Berghe, G.V., Krattenthaler, C.: An entry of Ramanujan on hypergeometric series in his notebooks. J. Comput. Appl. Math. 173(2), 239-246 (2005)

26. Prudnikov, A.P., Brychkov, Yu.A., Marichev, O.I.: More Special Functions. Integrals and Series, vol. 3. Gordon \& Breach, New York (1990)

27. Mitrinović, D.S.: Analytic Inequalities. Springer, New York (1970)

28. Agarwal, R.P., Elezović, N., Pečarić, J.: On some inequalities for beta and gamma functions via some classical inequalities. J. Inequal. Appl. 5, 593-613 (2005)

29. Dragomir, S.S., Agarwal, R.P., Barnett, N.S.: Inequalities for beta and gamma functions via some classical and new integral inequalities. J. Inequal. Appl. 5(2), 103-165 (2000)

30. Mitrinović, D.S., Pečarić, J.E., Fink, A.M.: Classical and New Inequalities in Analysis. Kluwer Academic, Dordrecht (1993)

31. Yeh, J.: Real Analysis, 2nd edn. World Scientific, Hackensack (2006)

32. Brychkov, Y.A.: Handbook of Special Functions: Derivatives, Integrals, Series and Other Formulas. CRC Press, Boca Raton (2008)

33. Qi, F:: Monotonicity results and inequalities for the gamma and incomplete gamma functions. Math. Inequal. Appl. $5(1), 61-67(2002)$

34. Qi, F., Cui, L.H., Xu, S.L.: Some inequalities constructed by Tchebysheff's integral inequality. Math. Inequal. Appl. 2(4), 517-528 (1999)

35. Qi, F., Guo, S.L.: Inequalities for the incomplete gamma and related functions. Math. Inequal. Appl. 2(1), 47-53 (1999)

36. Qi, F., Mei, J.Q.: Some inequalities of the incomplete gamma and related functions. Z. Anal. Anwend. 18(3), 793-799 (1999) 